\title{
The funding frontier
}

\author{
More and more private money is pouring into astronomical research and space exploration, and it's not all hype.
}

T he 7 January 2018 launch by SpaceX of a secret US government satellite known as Zuma - was not officially acknowledged as a success. Thus followed the rumours of a failed mission, with the blame bouncing between SpaceX, who claim that their Falcon 9 rocket performed as planned, and Northrop Grumman, the aerospace contractor that built the payload and the payload adaptor. But being a secret mission, perhaps everything did go according to plan and there is no confirmation just because. Either way, nobody is willing to say anything on record, citing "the classified nature of the payload".

That such public-private partnerships are on the rise is the more interesting story. With costs spiralling ever upwards, NASA for example, needs to outsource where it can, so it makes perfect sense to do so when the technology exists. This way, NASA can focus on the scientific mission and the bespoke technology required. Besides SpaceX, the only other US-based private contractor in the business of launching things for other people is United Launch Alliance, owned by Boeing and Lockheed Martin. (Blue Origin, founded by Jeff Bezos, is also in the space exploration business but on a smaller scale.)

Both SpaceX and Boeing have won NASA contracts to launch astronauts into space in 2018, though it seems 2019 will be more realistic. Seemingly unperturbed by the negative press from Zuma, SpaceX is preparing to 'test fire' the Falcon Heavy soon. This rocket has been designed to launch passengers into deep space, with Mars as a target. But the tests will be carrying mainly the reputation of SpaceX, whose reliability is in question after three setbacks prior to Zuma.

But leaving things to the private sector comes with a myriad of issues, not least those concerning regulations and accountability. Plus, they do care about good PR as well as the bottom line. There is a general worry that fundamental science will suffer.

So how do we best do science in the face of government austerity? In astronomy, telescopes don't come cheap. For instance, to fund new projects, the National Science Foundation is reducing its support of the Arecibo Observatory, among others, leaving them to look for new partners to contribute towards running costs. Fortunately, astronomy has a long history of patronage. The first 'research' observatories in Iraq and India in the 9th century were supported by their respective rulers to systematically map the movements of heavenly bodies. Royal patronage grew steadily but patchily from the 13th century, but the advent of international trade and empire-building, which depended heavily on a nation's navigational prowess, gave rise to a clutch of new observatories in the 17th century, including the Paris Observatory (backed by Louis XIV) and the Royal Observatory, Greenwich (commissioned by Charles II).

More recently, we have many examples of observatories founded by generous benefactors in the late 19th century: the Lick Observatory, the Smithsonian Astrophysical Observatory, the Lowell Observatory and more. And let's not forget the Sloan Digital Sky Survey from 2000, supported by the Alfred P. Sloan Foundation. Surveys are growing in importance and the data generated have led to innovations in the whole data-handling industry.

Today we have a new wave of billionaires who want to support research and development. They generally share a desire to make a positive difference, particularly in areas where they can take long-term risks, which can be harder to justify with public funding alone. The Gordon and Betty Moore Foundation, for instance, has committed US\$250 million towards the Thirty Meter Telescope. Bill Gates and the Charles and Lisa Simonyi Fund have donated US\$30 million towards the Large Synoptic Survey Telescope in Chile. Both of these telescopes are public-private partnerships that include governments.

There are also collaborations that do not involve governments directly, possibly reducing red tape and bureaucracy. The Giant Magellan Telescope, under construction in the Chilean Andes, was founded by a small number of universities and institutions, and will be the first of a generation of 'extremely large telescopes' to achieve first light in 2023. The Simons Foundation have put in US\$38.4 million for the Simons Observatory for studying the cosmic microwave background, also planned for Chile. And similarly, it has a small number of partner universities and institutions.

But this approach to fund bigger telescopes and larger surveys to answer questions on cosmology, dark matter and dark energy and the formation structure of the Universe, or to search for and characterize exoplanets and their atmospheres, is still quite 'safe' in terms of scientific funding topics. It would be more difficult to convince governments (and universities) to pump vast sums into looking for extraterrestrial life or reaching a planet beyond our Galaxy. And here, on the private end of the public-private partnership spectrum sits the Breakthrough Initiatives - Breakthrough Listen, Breakthrough Starshot, Breakthrough Message and Breakthrough Watch - funded by the Breakthrough Prize Foundation, founded by Yuri Milner. The goal is to answer the eternal question: are we alone?

By 'listening' and 'watching' they are searching for signs of life and compiling a list of targets for miniature light-powered nanocraft beyond the Solar System. Breakthrough Listen has committed US $\$ 100$ million (over 10 years) for SETI, buying time for extensive surveys on radio telescopes such as the Parkes and Green Bank observatories, and the optical Lick Observatory, and paying some researchers to collect and analyse data. Breakthrough Starshot aims to send a flyby mission to Alpha Centauri that would take 20 years instead of thousands. This initiative also has funding of US\$100 million for 5-10 years, and proposals for proofof-concept demonstrations of the light-sail technology have been reviewed and are soon to be funded.

Much of the hype surrounding the Breakthrough Initiatives sounds fantastical, but the science is underpinned by scientists. In fact, Breakthrough Listen has already contributed to research on fast radio bursts as well as the recent interstellar visitor 'Oumuamua. And that is the important point. As long as scientists are involved, even if the goal seems outlandish, interesting discoveries will be made along the way. We just need an enabler. Ultimately, whatever the funding model - public, publicprivate or private - science is advancing and we should embrace the resurgence of philanthropy.

Published online: 29 January 2018 https://doi.org/10.1038/s41550-018-0392-5 